\title{
A Systematic Review and Meta-Analysis of Molecular Biomarkers Associated with Early Neurological Deterioration Following Acute Stroke
}

\author{
Alexander J. Martin Christopher I. Price \\ NIHR Newcastle Biomedical Research Centre and Institute of Neuroscience, Newcastle University, Newcastle upon \\ Tyne, UK
}

\section{Keywords}

Acute stroke management · Biomarkers · Clinical outcome · Meta-analysis

\begin{abstract}
Background: Early neurological deterioration (END) following acute stroke is associated with poorer long-term outcomes. Identification of patients at risk could assist early monitoring and treatment decisions. This review summarised the evidence describing non-radiological biomarkers for END. Summary: Electronic searches from January 1990 to March 2017 identified studies reporting a blood/cerebrospinal fluid (CSF)/urine biomarker measurement within $24 \mathrm{~h}$ of acute stroke and at least 2 serial assessments of clinical neurological status ( $<24 \mathrm{~h}$ and $<7$ days). Out of 12,895 citations, 82 studies were included, mostly focusing on ischaemic stroke. Using higher neurological thresholds, the nweighted END incidence for ischaemic stroke was $11.9 \%$ (95\% Cl 11.4-12.4\%) and 18.6\% (17.9-19.2\%) for lower thresholds. Incidence decreased with advancing study publication year (Pearson r-squared 0.23 and 0.15 for higher and lower threshold studies). After classification into 3 broad categories, meta-analysis showed that biomarkers associated with increased END risk ( $\mathrm{n}$; fixed-effects mean difference; $95 \% \mathrm{Cl}$ ) were "metabolic" (glucose $[n=9,481 ; 0.90 \mathrm{mmol} / \mathrm{L}$; $0.74-1.06]$, glycosylated haemoglobin $[n=3,146 ; 0.33 \%$;
\end{abstract}

\section{KARGER}

() 2018 S. Karger AG, Basel

E-Mail karger@karger.com

www.karger.com/ced
$0.19-0.46]$, low-density lipoprotein $[n=4,839 ; 0.13 \mathrm{mmol} / \mathrm{L}$; $0.06-0.21]$, total cholesterol $[n=4,762 ; 0.21 \mathrm{mmol} / \mathrm{L} ; 0.11-$ $0.31]$, triglycerides $[n=4,820 ; 0.11 \mathrm{mmol} / \mathrm{L} ; 0.06-0.17]$, urea $[n=1,351 ; 0.55 \mathrm{mmol} / \mathrm{L} ; 0.14-0.96]$, decreasing albumin $[n=$ 513; $0.33 \mathrm{~g} / \mathrm{dL} ; 0.05-0.61]) ;$ "inflammatory and excitotoxic" (plasma glutamate $[n=688 ; 60.13 \mu \mathrm{mol} / \mathrm{L} ; 50.04-70.22]$, CSF glutamate $[n=369 ; 7.50 \mu \mathrm{mol} / \mathrm{L} ; 6.76-8.23]$, homocysteine $[n=824 ; 2.15 \mu \mathrm{mol} / \mathrm{L} ; 0.68-3.61]$, leucocytes $[n=3,766 ; 0.54 \times$ $\left.10^{9} / \mathrm{L} ; 0.34-0.74\right]$, high-sensitivity C-reactive protein $[n=$ 1,707;3.79 mg/L; 1.23-6.35]); and "coagulation/haematological" (fibrinogen $[n=3,132 ; 0.32 \mathrm{~g} / \mathrm{L} ; 0.25-0.40]$; decreasing haemoglobin $[n=3,586 ; 2.38 \mathrm{~g} / \mathrm{L} ; 0.15-4.60])$. Key Messages: Declining incidence of END may represent improving care standards; however, it remains a frequent occurrence. Although statistical associations exist between biomarkers and an increased risk of END, the most promising still need prospective evaluation to determine their additional value relative to baseline radiological and clinical characteristics.

(c) 2018 S. Karger AG, Basel

\section{Introduction}

Early neurological deterioration (END) describes the worsening of symptoms in the hours and days following acute stroke and is associated with worse outcomes [1]. Although it may reflect natural progression of the

Christopher I. Price, MD 
underlying pathophysiological process, END is not inevitable and occurs less often when care is provided within a formal stroke unit setting [2]. Associations include age, diabetes, cerebral oedema, haematoma expansion (particularly in the context of hypertension), haemorrhagic conversion of infarction with or without reperfusion treatment and systemic physiological disturbances such as blood pressure variability and pyrexia [1-5].

Variations in case-mix and END definitions have produced wide estimates of incidence, with a previous review reporting frequencies of $2.2-37.5 \%$ at $24 \mathrm{~h}$ since onset [1]. Fewer patients will be labelled with END at a higher clinical threshold than a lower threshold. It may be also expected that the probability of END will increase with longer time windows between first and second measurements of clinical status. By any definition, prediction of END risk at the point of admission may identify patients in need of additional monitoring, investigations and a lower threshold for clinical intervention.

Clinical and radiological associations with END have already been described in detail [1]. A molecular biomarker is particularly attractive for predicting END as it could be developed into a point-of-care test applied at the beginning of the patient care pathway to provide additional subclinical information about prognosis. A recent horizon scanning review of stroke biomarkers identified associations between END and markers of excitotoxicity (glutamate and glycine), pro-inflammatory cytokine interleukin (IL)-6 and fluorescent molecular peroxidation products [3]. However, it did not consider information about possible biomarkers contained within reports from large clinical studies or perform meta-analysis. In view of the increasing focus upon stratification of stroke patients for different emergency treatments and levels of monitoring intensity, we undertook an in-depth review across a broad range of clinical studies to describe END frequency, categorize molecular biomarkers into a simple taxonomy and consider their potential as predictors for END risk, with meta-analysis when possible.

\section{Methods}

Medline and Embase were searched from January 1990 to March 2017 for interventional or observational studies reporting a blood, cerebrospinal fluid (CSF) or urine biomarker measurement early after acute ischaemic and/or haemorrhagic stroke, including at least 2 serial assessments of clinical neurological status (baseline $<24 \mathrm{~h}$ and repeated within 7 days). The protocol was registered with the PROSPERO review database:IDCRD42017060167.
The search strategy is described in online supplement 1 (for all onlinesupplmaterial,seewww.karger.com/doi/10.1159/000495572). Only studies written in English were included. Studies were initially screened by title and abstract. Full text versions were then sought, but authors were not contacted. Data items (online suppl. 2) were extracted using a pre-defined proforma including setting, population, participant demographics and baseline characteristics, biomarkers, time of biomarker measurement and author definitions for END, ischaemic END (IS-END) and END following haemorrhagic stroke (ICH-END). Unclear study descriptions were reviewed by both authors. Studies were excluded that specifically included subarachnoid haemorrhage or other vascular malformations and from settings that report highly unusual clinical stroke care practices (e.g., brain imaging was not routinely performed). Due to the heterogeneity of studies and information available, no formal assessment of risk of bias was possible.

Review Manager (Revman) version 5.3 [4] was used for metaanalysis of individual biomarkers where a mean and SD were reported for each group. Standard conversions were made to common units. A fixed effects model was used and mean difference (95\% CI) is reported. No additional analyses were performed. If a biomarker was described by only 1 study, the results are presented within the meta-analysis results if the data were normally distributed. All studies reporting data which were not normally distributed, and those which could not be combined because of lack of information about the END definition, are described but are not included in the meta-analysis.

\section{Results}

Online supplement 3 shows the Preferred Reporting Items for Systematic Reviews and Meta-Analyses [5] flowchart of studies; 86 were included from 12,895 initial citations. Table 1 summarises studies grouped by authordefined neurological thresholds for END.

Using a higher neurological threshold, 35 IS/mixed studies had n-weighted END incidence of 11.9\% (95\% CI 11.3-12.4\%), whereas 43 lower-threshold studies had incidence of $18.0 \%$ (95\% CI 17.4-18.7\%; online suppl. 4). Three higher-threshold ICH studies had an incidence of $12.3 \%$ (95\% CI 9.2-15.4\%), and a single lower threshold ICH study [6] had an incidence of $23.0 \%$ (95\% CI $17.9-$ 28.1\%; online suppl. 4).

There was a trend of declining END incidence with advancing study publication year, which persisted when stratified by time window and definition (Fig. 1,2).

Biomarker data are summarised in 3 categories: (a) metabolic (Table 2); (b) inflammatory and excitotoxic (Table 3) and (c) coagulation and haematological (Table 4). Studies reporting non-parametric biomarker data, results based on a threshold and biomarkers described in single studies only were unsuitable for meta-analysis and are tabulated separately in online supplement 5, 6 and 7 
Fig. 1. Incidence of END against study publication year and Pearson r-squared values, stratified by clinical assessment interval. END, early neurological deterioration.

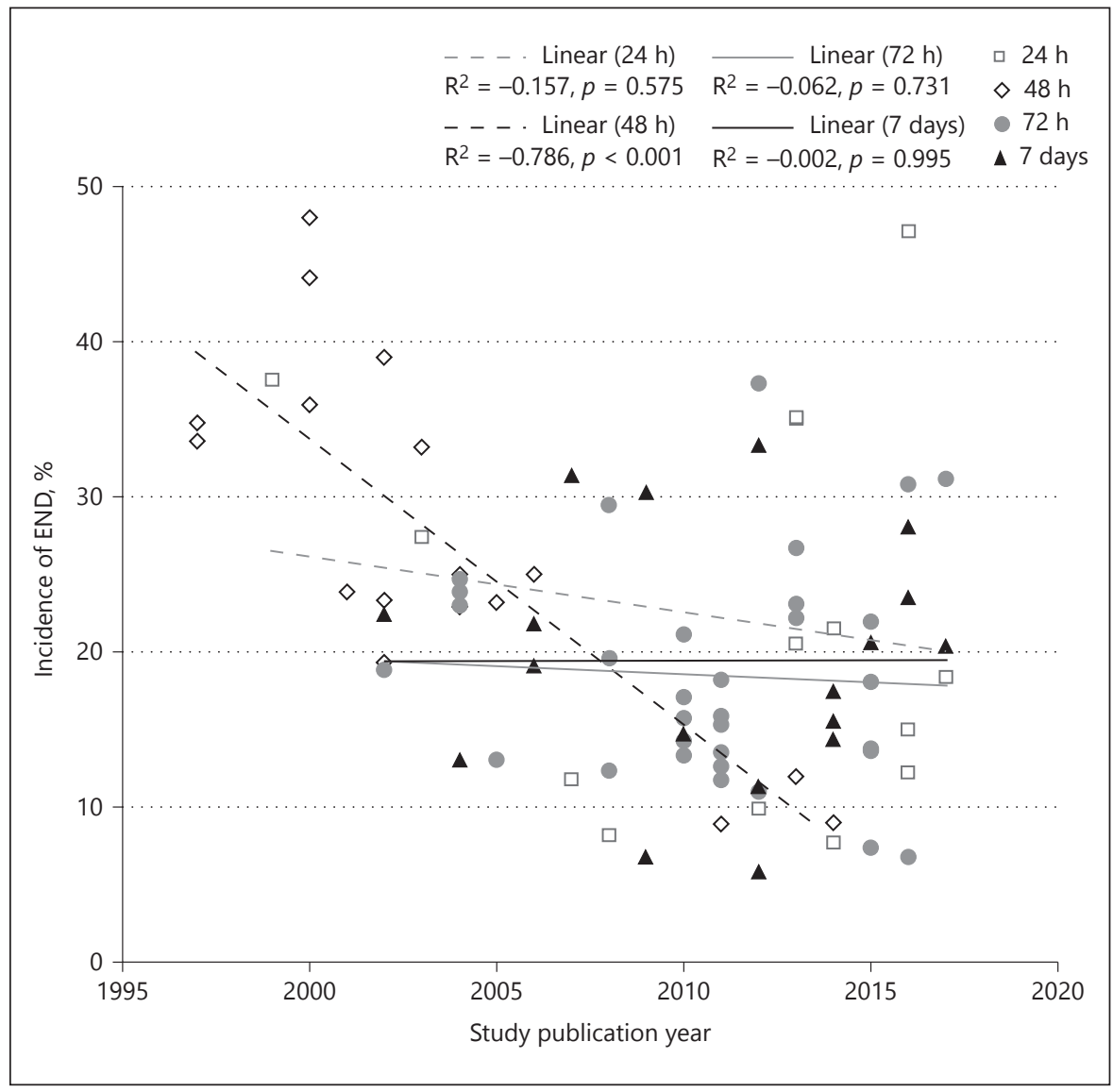

Table 1. Count of studies by END definition and time

\begin{tabular}{|c|c|c|c|c|c|}
\hline \multirow[t]{2}{*}{ END definition } & \multicolumn{5}{|c|}{ END time } \\
\hline & $24 \mathrm{~h}$ & $48 \mathrm{~h}$ & $72 \mathrm{~h}$ & 7 days & total \\
\hline \multicolumn{6}{|l|}{ Lower threshold } \\
\hline NIHSS $\geq 1$ & 1 & 1 & 5 & 3 & 10 \\
\hline NIHSS $\geq 2$ & 3 & & 5 & 10 & 18 \\
\hline CSS $[8] \leq-1$ & & 16 & 2 & & 18 \\
\hline SSS [9] $\leq-2$ & & & 1 & & 1 \\
\hline \multicolumn{6}{|l|}{ Higher threshold } \\
\hline NIHSS $\geq 3$ & & & 3 & & 3 \\
\hline NIHSS $\geq 4$ & 10 & 2 & 7 & 5 & 25 \\
\hline SSS $\leq-3$ & & 1 & & & 1 \\
\hline $\mathrm{GCS} \geq 3$ & & & 1 & & 1 \\
\hline EPSS criteria $[10]^{*}$ or modified & 1 & & 9 & & 10 \\
\hline Total & 15 & 20 & 33 & 18 & 86 \\
\hline
\end{tabular}

* European Progressing Stroke Study (EPSS) criteria (SSS $\leq-3$ drop speech or $\leq-2$ level of consciousness, arm, hand, leg or death).

CSS, Canadian Stroke Scale; SSS, Scandinavian Stroke Scale; GCS, Glasgow Coma Scale; END, early neurological deterioration. 
Fig. 2. Incidence of END against study publication year and Pearson r-squared values, stratified by neurological threshold used to define END. END, early neurological deterioration.

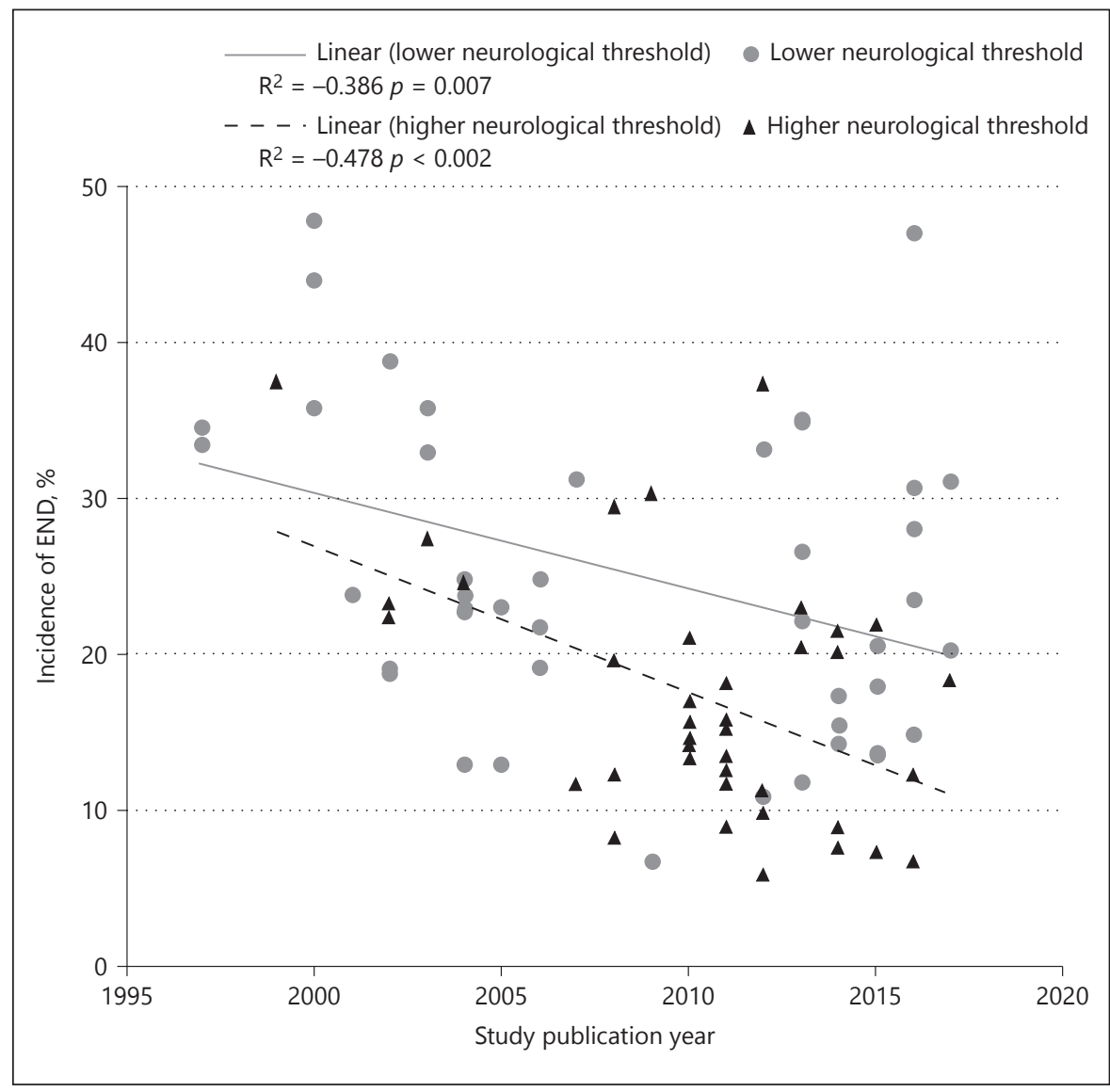

and are referenced by their entry in each Supplement. Citations in bold font concur with the output from the relevant biomarker meta-analysis.

\section{Metabolic Biomarkers}

Glucose was significantly higher with IS-END [2, 7-36] and ICH-END [37], which may have numerous explanations including poorer collateral flow, endothelial damage, increased oxidative stress and lactate, haemorrhagic transformation and dysregulation of glucose energy metabolism [38]. This was mirrored in glycosylated haemoglobin A1c, a marker of chronic raised glucose, also predicting END [9, 23, 25, 26, 29, 30, 35, 39] [5K]. One study stratified the effect of glucose by the presence of diabetes mellitus, finding elevated glucose level was associated with END in non-diabetics [38].

Raised total cholesterol, low-density lipoprotein, triglycerides and triglyceride/high-density lipoprotein ratio were predictive of IS-END; however, lower levels of these predicted ICH-END [9, 23, 25, 26, 35, 39-45] [5M, 5S-U]. This concurs with current understanding about cholesterol and the risk of IS/ICH. Hypercholesterolaemia is well described as a modifiable risk factor for IS and lipid-lowering agents are used in stroke prevention. Conversely, possibly because a degree of cholesterol deposition plays a role in maintaining the arterial wall, risk of rupture and haemorrhage is lower in patients with higher total cholesterol [46]. Furthermore, post-hoc analysis of the Stroke Prevention by Aggressive Reduction in Cholesterol Levels trial showed early treatment post-IS with high-dose lipid-lowering agents increased ICH risk [47]. There was no association between high-density lipoprotein and END [9, 23, $29,35,40-44][5 \mathrm{~L}]$.

Increased serum albumin appeared to reduce the risk of END. The included studies did not suggest why [25, 26], however, a 2004 study found that higher albumin decreased the risk of poor outcome at 3 months indicating a possible link between pre-stroke health and END [48]. A higher ratio of CSF: serum albumin predicted END as a marker of albumin influx into the CSF following bloodbrain barrier dysfunction in stroke [49]. 
Table 2. Metabolic biomarkers meta-analysis

\begin{tabular}{|c|c|c|c|c|}
\hline Biomarker (stroke type), unit & Studies & Participants & Effect & Appendix \\
\hline Glucose, $\mathrm{mmol} / \mathrm{L}$ & 31 & 9,781 & $0.90(0.74$ to 1.06$)$ & 8 \\
\hline Ischaemic & 30 & 9,669 & $0.89(0.74$ to 1.05$)$ & \\
\hline Glycosylated HbA1c (IS), \% & 9 & 3,146 & $0.33(0.19$ to 0.46$)$ & 9 \\
\hline $\mathrm{HDL}, \mathrm{mmol} / \mathrm{L}$ & 9 & 4,597 & $0.02(-0.01$ to 0.04$)$ & 10 \\
\hline Ischaemic & 9 & 4,537 & $0.02(-0.01$ to 0.05$)$ & \\
\hline Ischaemic & 14 & 5,416 & $0.13(0.06$ to 0.21$)$ & \\
\hline Haemorrhagic & 1 & 60 & $-0.20(-0.73$ to 0.33$)$ & \\
\hline Total cholesterol, mmol/L & 12 & 4,822 & $0.19(0.10$ to 0.29$)$ & 12 \\
\hline Ischaemic & 12 & 4,762 & $0.21(0.11$ to 0.31$)$ & \\
\hline Haemorrhagic & 1 & 60 & $-0.41(-1.00$ to 0.18$)$ & \\
\hline ALT (IS), IU/L & 4 & 992 & $-0.14(-2.56$ to 2.27$)$ & 15 \\
\hline AST (IS), IU/L & 4 & 992 & $-5.14(-7.03$ to -3.26$)$ & 16 \\
\hline Sodium (IS), $\mathrm{mmol} / \mathrm{L}$ & 3 & 838 & $-0.58(-1.21$ to 0.04$)$ & 17 \\
\hline Potassium (IS), $\mathrm{mmol} / \mathrm{L}$ & 3 & 838 & $-0.09(-0.18$ to 0.00$)$ & 18 \\
\hline Creatinine (IS), $\mu \mathrm{mol} / \mathrm{L}$ & 4 & 1,034 & $0.13(-5.14$ to 5.40$)$ & 19 \\
\hline eGFR (IS), $\mathrm{mL} / \mathrm{min} / 1.73 \mathrm{~m}^{3}$ & 3 & 915 & $-0.53(-5.34$ to 4.28$)$ & 20 \\
\hline Urea (IS), $\mathrm{mmol} / \mathrm{L}$ & 5 & 1,351 & $0.55(0.14$ to 0.96$)$ & 21 \\
\hline $\mathrm{UA}$ (IS), $\mu \mathrm{mol} / \mathrm{L}$ & 3 & 874 & $-13.18(-32.94$ to 6.58$)$ & 22 \\
\hline
\end{tabular}

IS, ischaemic; HbA1c, haemoglobin A1c; HDL, high-density lipoprotein; LDL, low-density lipoprotein; ALT, alanine aminotransferase; AST, aspartate transaminase; UA, uric acid.

Table 3. Inflammatory and excitotoxic biomarkers

\begin{tabular}{|c|c|c|c|c|}
\hline $\mathrm{CRP}, \mathrm{mg} / \mathrm{L}$ & 5 & 1,481 & $3.02(0.41$ to 5.63$)$ & 23 \\
\hline Ischaemic & 4 & 1,369 & $0.00(-3.88$ to 3.88$)$ & \\
\hline Haemorrhagic & 1 & 112 & $5.50(1.98$ to 9.02$)$ & \\
\hline hsCRP (IS), mg/L & 5 & 1,707 & $3.79(1.23$ to 6.35$)$ & 24 \\
\hline D-dimer, mg/L & 4 & 563 & $0.07(-0.06$ to 0.20$)$ & 25 \\
\hline Glutamate (CSF; IS), $\mu \mathrm{mol} / \mathrm{L}$ & 2 & 369 & $7.50(6.76$ to 8.23$)$ & 26 \\
\hline Glutamate (plasma), $\mu \mathrm{mol} / \mathrm{L}$ & 5 & 688 & $60.13(50.04$ to 70.22$)$ & 27 \\
\hline Mixed & 1 & 36 & $-4.00(-18.93$ to 10.93$)$ & \\
\hline Ischaemic & 4 & 652 & $113.99(100.31$ to 127.68$)$ & \\
\hline Glycine (plasma; IS), $\mu \mathrm{mol} / \mathrm{L}$ & 3 & 277 & $6.75(-0.62$ to 14.11$)$ & 28 \\
\hline Homocysteine (IS), $\mu \mathrm{mol} / \mathrm{L}$ & 2 & 824 & $2.15(0.68$ to 3.61$)$ & 29 \\
\hline
\end{tabular}

CRP, C-reactive protein; hsCRP, high sensitivity C-reactive protein; CSF, cerebrospinal fluid. 
Table 4. Coagulation and haematological biomarkers

\begin{tabular}{lccc}
\hline Biomarker (stroke type), unit & Studies & Participants & Effect \\
\hline APTT, s & 5 & 3,051 & $-0.18(-0.78$ to 0.42$)$ \\
$\quad$ Ischaemic & 4 & 2,939 & $-0.32(-0.94$ to 0.30$)$ \\
$\quad$ Haemorrhagic & 1 & 112 & $2.00(-0.43$ to 4.43$)$ \\
Fibrinogen, g/L & 15 & 3,132 & $0.32(0.25$ to 0.40$)$ \\
$\quad$ Ischaemic & 13 & 2,786 & $0.29(0.21$ to 0.36$)$ \\
$\quad$ Haemorrhagic & 2 & 346 & $0.99(0.67$ to 1.32$)$ \\
Haematocrit (IS), $\%$ & 5 & 2,865 & $-0.84(-1.74$ to 0.07$)$ \\
Haemoglobin, g/L & 9 & 3,964 & $-1.05(-3.02$ to 0.92$)$ \\
Ischaemic & 7 & 3,586 & $-2.38(-4.60$ to -0.15$)$ \\
Haemorrhagic & 2 & 378 & $-0.06(-0.12$ to -0.00$)$ \\
INR (IS) & 3 & 2,924 & $1.94(-5.20$ to 9.08$)$ \\
Platelets, $\times 10^{9} / \mathrm{L}$ & 9 & 4,017 & $3.22(-4.30$ to 10.74$)$ \\
Ischaemic & 8 & 3,905 & $-9.90(-32.78$ to 12.98$)$ \\
Haemorrhagic & 1 & 112 & 33 \\
\hline
\end{tabular}

APTT, activated partial thromboplastin time; INR, International normalised ratio; IS, ischaemic.

The liver enzyme aspartate transaminase (AST) metabolises glutamate. Higher levels of liver enzyme AST (glutamate-oxaloacetate transaminase), which metabolises glutamate, were associated with lower incidence of END $[9,11,25,26]$. Glutamate may be excitotoxic in stroke [50]; therefore, AST may be neuroprotective by reducing metabolic activity [11]. However, alanine transaminase (glutamate pyruvate transaminase) was not significantly different in END. Increased bilirubin was associated with END in 1 study [5F].

Further results from studies that could not be combined into meta-analysis are shown in online supplement 5.

END was associated with higher blood urea nitrogen: creatinine ratio [5H-I], urine-specific gravity [5Z], urea $[9,22,25,26,29]$ and urinary albumin:creatinine ratio [5V-X], markers of dehydration/impaired renal function. Of 2 studies of plasma osmolality/osmolality [5Q-R], 1 found an association with END, although the 1 direct study of plasma viscosity did not reach significance [7Q]. Patients with dehydration may suffer END due to increased plasma viscosity and/or lower cerebral perfusion, but these biomarkers for renal function and fluid status may also indicate co-morbidities and limited physiological reserve.

Uric acid (UA), an antioxidant, was found in 1 study to decrease with time following stroke and a greater decrease by day 7 was associated with END [5Y], possibly because higher baseline UA is associated with a greater vascular risk.

Biomarkers to Predict END after Stroke
Plasma adrenomedullin was higher in ICH-END, but in a single study did not improve the predictive value of the NIHSS score [5B].

Creatinine phosphokinase was associated with ICHEND in 1 study; however, this was only narrowly significant $(p=0.043)$ in a study where $\sim 60$ variables were analysed and no adjustment for multiple comparisons was made [5I].

Adiponectin [5A], alkaline phosphatase [5D], alanine aminotransferase $[9,11,25,26]$, aspartate [5E], acute decrease in magnesium [5N], methionine [5O], sodium [9, $22,29]$, potassium $[9,22,29]$, creatinine $[9,22,26,29]$, estimated glomerular filtration rate $[10,18,29][5 \mathrm{~J}]$ and UA $[12,25,26]$ showed no association with END.

\section{Inflammatory and Excitotoxic Biomarkers}

Excitotoxic amino acids are associated with damage in stroke, particularly glutamate [50], which induces depolarisations around the infarct that disrupt ionic homeostasis, increasing infarct volumes [51]. In meta-analysis, glutamate was associated with END in CSF [24, 52], although this was not corroborated by a further small study [6O]. Plasma glutamate was also associated with IS-END $[8,11,52,53][6 \mathrm{~N}]$. Cysteine $[6 \mathrm{H}]$, homocysteine $[23,35]$ $[6 \mathrm{~S}]$ and CSF glycine [6Q, 6R] were also associated with END in at least 1 study. Phosphorylated axonal subunit$\mathrm{H}$, a marker of axonal injury, was raised in $\mathrm{ICH}$; however, it did not improve on the PPV of NIHSS alone [6AI]. Glutamine $[6 \mathrm{P}]$, proline $[6 \mathrm{AJ}]$ and taurine $[6 \mathrm{AS}]$ were not associated with END. 
Markers of inflammatory immune response were also raised in END, which may suggest ongoing inflammation or an increased risk associated with underlying infection. Standard C-reactive protein (CRP) was significantly different in ICH [37] and in some studies of IS [6G]. However, high-sensitivity CRP (hsCRP) predicted END in IS $[12,23,41,43,54]$, which is supported by studies showing that raised hsCRP is also associated with IS risk [55]. Leucocyte count was elevated in END in IS [7-9, 11, 16, 17, $22,25,26,29,35,36,39,45,53,56,57]$ [AD] and ICH [6, 37]. Neutrophil count was raised in END in $1 \mathrm{ICH}$ study [6AF], as were segmented neutrophils in IS [6AM]. (s) ICAM-1, which is responsible for neutrophil adhesion [36], was associated with END in 2 studies [6U, 6AO]. Pro-inflammatory cytokines IL-6 [6Z] and TNFa (in plas$\mathrm{ma}$ and CSF) [6AT-U] were raised in END. TNFa receptors sTNF-R1/R2 were not associated with END [6APAQ].

There were several notable findings amongst studies which could not be combined in the meta-analysis (online suppl. 6).

Molecules with a role in the inflammatory response included 15-deoxy prostaglandin J2, which appeared to be neuroprotective against END in atherothrombotic infarcts, but not in other infarct types [6A-B], whereas elevated beta-defensin $2[6 \mathrm{D}]$ and soluble IL-4 receptor [6Y] improved the prediction of END over clinical models.

Nitric oxide (NO) has both neuroprotective and neurotoxic roles in brain injury. NO-metabolite predicted END independent of CSF glutamate levels [6AG], whereas $\mathrm{L}$-arginine, a substrate for $\mathrm{NO}$ generation and potential marker of NO-mediated brain damage, was lower in patients with END [6AA-AB].

In a study of monocyte subsets, an increased proportion of CD14 ${ }^{\text {high }} \mathrm{CD} 16$ - monocytes, was associated with END by 7 days, potentially because this meant fewer CD$14{ }^{\text {high }} \mathrm{CD} 16+$ monocytes were present $[6 \mathrm{E}]$. The latter produce IL-10, which has been suggested to have a beneficial anti-inflammatory effect in stroke [57]. However, while a study of numerous cytokines and receptors found a significant association between IL-10 and END, it was concluded that it did not predict END better than clinical assessment alone $[6 \mathrm{~V}]$. The study found no association with IL-1B or IL-1RA [6W-X] and END. In a study focussing upon acute penetrating artery stroke, low baseline CD34+ cells, markers of endothelial function, were associated with END $[6 \mathrm{~F}]$.

In a CSF study of monoaminergic neurotransmitters, only elevated 5-hydroxyindoleacetic acid was associated with END [6C]. Norepinephrine [6AH], 5-hydroxyin- doleacetic acid [6C], homovanillic acid [6T] and 3,4-dihydroxyphenylacetic acid [6I] were not.

Two indicators of blood-brain barrier compromise were associated with END:CSF:serum albumin ratio [5C] and change in matrix metalloproteinase-9 [6AE]. Fluorescent molecular peroxidation products, markers of oxidative damage hypothesised to promote blood-brain barrier degradation, were significantly associated with END following thrombolysis [6L].

Cysteine [6H], gamma-aminobutyric acid [6M], plasma glycine [6R], p-selectin [6AK], SE-selectin [6AN], segmented and band leucocytes [6AL] and sVCAM-1 [6AR] were not significantly different in END.

A single study showed a non-significant trend towards elevated CSF lactate indicating a higher risk of END $[6 \mathrm{AC}]$. Although lactate is generally a useful marker of ischaemia, the low capacity for anaerobic metabolism within neurones may result in the ability to detect only large volumes of vulnerable tissue.

\section{Coagulation and Haematological Biomarkers}

Pro-coagulation factors fibrinogen (In IS $[7,8,11,15-$ $17,28,31,35,36,45,53,56]$ [7M]; in ICH $[6,37])$ and factor VIII [7I] were associated with END, as was INR, a measure of the intrinsic coagulation pathway $[29,36,42]$ (Table 4). A greater $72 \mathrm{~h}$ decrease in procarboxypeptidase $\mathrm{U}$, which inhibits fibrinolysis, predicted END, which was hypothesised to be utilised by thrombus growth [7T]. Measures of endogenous anti-coagulants protein C [7B], antithrombin-III [7E] and thrombin-antithrombin III complex [7Y] did not predict END.

Aspirin non-responsiveness may also play a role in END. Platelet reactivity to aspirin (ARU) at baseline was not associated with END; however, a high degree of change in ARU was associated with END [7A]. Poor response to aspirin measured by PFA-100s, a platelet aggregation assay, was also associated with END [7D].

Lower haemoglobin was associated with END in IS [9, $22,25,29,42]$, which may suggest patients with reduced oxygen-carrying capacity are at greater risk.

Further studies found significant associations which could not be combined in meta-analysis (online suppl. 7). Both CSF and plasma ferritin were associated with END independently of ultimate infarct volume and were hypothesised to be related to excitotoxic mechanisms in stroke; however, only the CSF ferritin finding was replicated in a later study [7K-L]. Neither CSF nor plasma iron were associated with END [7O-P].

Higher von Willebrand factor was associated with END [7AA]. In the same study, 3 markers of thrombin 
Table 5. Summary of the biomarkers showing an association with END in the meta-analysis

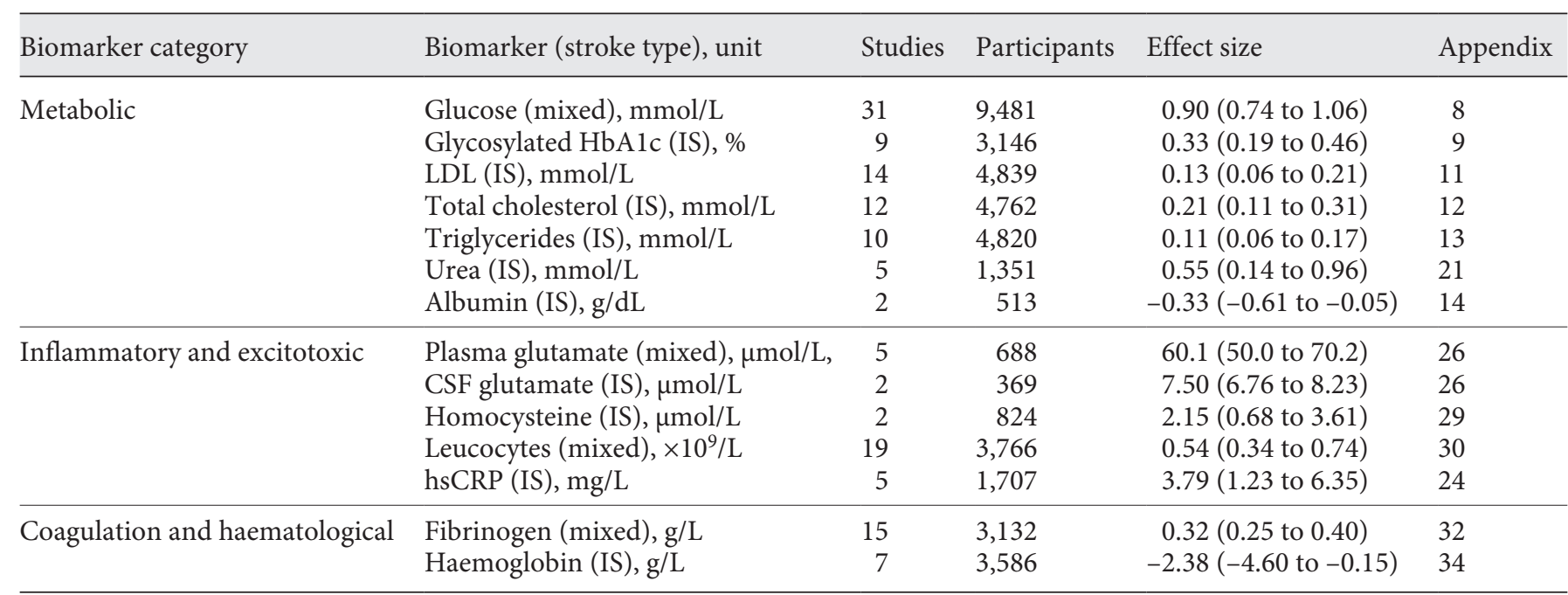

IS, ischaemic stroke, mixed, ischaemic and haemorrhagic stroke; HbA1c, haemoglobin A1c; CSF, cerebrospinal fluid; hsCRP, highsensitivity c-reactive protein; LDL, low-density lipoprotein; END, early neurological deterioration.

generation (prothrombin F1 +2 [7V], fibrin d-dimer [7F] and thrombin-antithrombin complexes [7Y]) were associated with END on univariate analysis. Six studies showed increased D-dimer associated with END, 3 of which reported the associated as significant.

Activated partial thromboplastin time [7C], factors VIIc and VIII(c) [7H-J], haematocrit [7N], platelets [7R], prolyl carboxypeptidase [7S], prothrombin fragment $1+$ 2 [7U], prothrombin time [7V], soluble fibrin monomer $[7 \mathrm{~W}]$ and thrombin time [7X] were no different with END.

\section{Discussion}

Depending upon the threshold used, IS-END incidence ranged from 11.9 (higher threshold definition) to $18.6 \%$ (lower threshold definition), indicating that it remains a significant clinical challenge. As only 4 studies focussed upon ICH in isolation, there is limited data about ICH-END incidence in relation to biomarkers, but amongst the 3 using a higher threshold, 12.2\% deteriorated, comparable to ischaemic stroke. In cohort studies, END rates up to $30 \%$ have been reported following ICH [58], but differences are likely to reflect whether the clinical setting is a stroke unit without pre-selection or a specialist neurosurgical unit. Overall, incidence of END fell with advancing study publication year, independently of neurological threshold and time window. This may re- flect improvements in stroke care including reperfusion treatments and/or changes in case-mix.

Meta-analysis identified a large range number of biomarkers, both routinely performed and novel. To indicate those with greater potential to influence future research and clinical care, biomarkers significantly associated with END following pooling of more than 1 study are summarised in Table 5.

The likely explanations for these associations are diverse, and a direct link to mechanisms for END cannot be inferred. Some of the biomarkers described have been considered by trialists as contributors themselves towards the process of neuronal death, but intervention has not consistently shown improved outcomes, for example, insulin for blood glucose lowering [59]. However, there is consistent trial evidence that pro-active assessment for pyrexia, swallowing difficulties and dehydration can reduce dependency, possibly through avoidance of END for some patients. It is therefore not surprising that infection (e.g., leucocytes and hsCRP) and fluid status/renal function biomarkers (urea, albumin) may identify patient groups particularly at risk. The link to END may be through the physiological consequences of infection and dehydration such as increased plasma viscosity and fluctuations in cerebral perfusion.

Other biomarkers may reflect the volume of subclinical cerebral tissue injury during acute stroke and hence indicate a greater probability of further clinical progression, particularly those molecules which are specific to 
neuronal tissue or involved in neurotransmission. For example, glutamate, which is also implicated in the progression of cellular death through excitotoxicity $[51,53]$, was associated with END when measured in CSF and plasma. We did not identify any studies which related these biomarkers to advanced imaging techniques such as MRI or CT perfusion, which could provide mechanistic evidence and indicate their potential for further development as rapid near-patient tests. There is already good evidence that perfusion mismatch indicates patients at risk of deterioration [60], which can be averted by reperfusion therapy [61], but a point of care biomarker with sufficient accuracy to alter clinical care is likely to have wider applicability and availability for the global stroke population.

In line with growing evidence that inflammation is key to the development of atherosclerosis and plaque rupture, associations have been shown between biomarkers implicated in inflammatory pathways and END. These may reflect the severity of pre-existing atheroma and so indicate poorer local perfusion and/or collateral supply [62]. Elevated haemoglobin A1c, increased low-density lipoprotein, total cholesterol and triglycerides may also indicate a greater burden of background atheroma and vulnerability to tissue ischaemia. The relationship between haematological biomarkers and END is likely to be more direct and immediate. Fibrinogen, a pro-coagulant factor, may reflect the probability of large, thrombin-rich clot formation and progression, whereas impaired penumbral oxygen delivery may explain the association between END and reduced haemoglobin.

A recent review identified numerous challenges when developing biomarkers for stroke diagnosis which are pertinent to predicting END [3]. These include the non-specificity of biomarkers to brain tissue and the inability of molecules to cross the blood-brain barrier. Simultaneous measurement of multiple biomarkers in a single panel has been proposed, combined with recent developments in genomics and proteomics, approaches which have not yet been taken in END. However, as reported by the StrokeChip study following evaluation of an assay panel for stroke diagnosis, many biomarkers are not independent of one another and may not be significantly better than clinical judgement and radiological data [63]. Amongst investigations, which are already routinely available clinically, the results of the meta-analysis suggest that it would be reasonable to increase monitoring intensity for those acute stroke patients where venipuncture reveals a low haemoglobin or elevated glucose, glycosylated haemoglobin, urea, osmolality, CRP, ESR or leucocytes.

\section{Limitations}

This review included only summary results from English language studies indexed in Medline and Embase, and authors were not contacted for data. Most articles provided insufficient information to permit assessment of bias. For example, many studies reported that the biomarker results were concealed from the END assessor, but it was unclear whether they were aware of other results which could cause unblinding such as clinical brain imaging. Formal quality assessment of the included studies was not performed as interpretation would have been difficult due to their early stage, extreme methodological variability and the lack of information presented. Sample size estimations were rarely reported. Despite proven associations with patient outcomes, studies did not report other clinical or radiological data, which could assist with exploration of mechanisms for biomarker responses and their clinical utility.

A significant limitation of this study is the lack of data on important confounding factors. For studies including patients both with and without reperfusion treatment, individual patient reperfusion status was not sought. It is likely that reperfusion treatment will change the magnitude of the association between END and any biomarker. In clinical practice, it is possible that a biomarker reading performed prior to reperfusion treatment, which indicates a greater risk of END may also be used in a treatment decision as it may represent the volume of threatened brain tissue.

Additionally, although timing of biomarker measurement was sometimes reported, these data were not available for individual patients. Very early or late sampling for biomarker identification of stroke may lead to false negative cases: for example, Rozanski et al. [64] pre-hospital study of Glial Fibrillary Acidic Protein as a biomarker of ICH, with a median onset-to-sampling time of 63 min, had poorer sensitivity than hospital-based studies (median onset-to-sampling time $>120 \mathrm{~min}$ ). The same may apply to the use of biomarkers to detect END as levels may not yet have risen or have already fallen.

Biomarker utility may also vary by stroke subtype. This reflects the increasing evidence that different mechanisms may be responsible for small vessel stroke (e.g., inflammation [65]) and large vessel stroke (e.g., atrial fibrillation). In future clinical practice, it may be necessary to apply different biomarkers for END according to the stroke subtype and early indicators of the possible aetiology such as ECG appearances. Although some studies analysed END incidence by clinical vascular territory or ischaemic stroke origin, few studies (e.g., Matsumoto et al. [28]) explicitly broke down biomarker performance by 
these subgroups or analysed a specific subgroup, and so this level of detail could not be included as a sensitivity analysis. A further complication in understanding such data would be the variable probability of deterioration across different stroke subtypes. An individual patient data meta-analysis broken down by clinical, radiological and test characteristics would be the most appropriate way to address this, but currently would be very challenging and future studies should report key clinical and confounding factors using standardised approaches and units to enable reliable pooling of subgroups.

\section{Recommendations}

The biomarkers identified by this review, which have demonstrated strong associations with END in more than 1 cohort, have the potential to be clinically useful, but most were derived by exploratory retrospective analysis further independent prospective studies are required to prove diagnostic utility. These should include reports of standardised clinical and radiological data, such as the Alberta Stroke Program Early CT Score [66] for ischaemic stroke and haematoma volume for ICH [58]. Other novel biomarkers with a promising pathophysiological basis were considered by single studies, but have not been further developed. Significant investment is needed if affordable and practical tests are to become available for integration into effective clinical monitoring and response protocols.

As END remains a clinical entity with various definitions, standardisation is necessary to allow meaningful comparison and combination of future studies. In the meantime, authors should determine a priori which definition is to be used. The timing of biomarker sampling and assessment of END should be at clinically important intervals, for example, during thrombolysis/thrombectomy windows. Other factors that may influence END incidence (e.g., time from onset and thrombolysis/thrombectomy treatment) should be collected within studies that are large enough to allow meaningful analysis of multiple variables.

There are emerging diagnostic biomarkers with high specificity for neurological tissue, which could also be considered by future studies of END [67]. For example, plasma glial fibrillary acidic protein shows $>90 \%$ specificity as a biomarker for haemorrhagic stroke, and concentrations are positively correlated with the risk of subsequent clinical deterioration [64]. Neurofilaments are released during neuroaxonal injury [68], including following traumatic brain injury [69], but are also elevated in active cerebral small vessel disease [70] and acute ischaemic stroke [71], particularly in those with higher severity of primary injury [72] and recurrent ischaemic lesions [71]. These markers may prove a valuable avenue of investigation for further studies of END. Whether alone or in combination, and informed by initial brain imaging, the high specificity of these biomarkers may provide valuable information about the subclinical volume of tissue injury and predict END. However, further studies are required.

\section{Conclusions}

Although the incidence of END following acute stroke may be decreasing, it remains a common occurrence. This systematic review has identified biomarkers associated with END in predominantly exploratory studies or from incidental observation during clinical trials. Their value in relation to key clinical and radiological characteristics is unclear, and none can yet be recommended for use. Further studies are required for validation and development of clinically useful tests, which stratify patients into higher- and lower-risk groups, and ultimately improve clinical outcomes through END prediction.

\section{Disclosure Statement}

No conflicts of interest to declare by any author.

\section{Funding Sources}

This research was not supported by any funding sources.

\section{References}

1 Seners P, Turc G, Oppenheim C, Baron JC: Incidence, causes and predictors of neurological deterioration occurring within $24 \mathrm{~h}$ following acute ischaemic stroke: a systematic review with pathophysiological implications. J Neurol Neurosurg Psychiatry 2015;86:8794.

2 Roquer J, Rodriguez-Campello A, Gomis M Jimenez-Conde J, Cuadrado-Godia E, Vivanco R, et al: Acute stroke unit care and early neurological deterioration in ischemic stroke. J Neurol 2008;255:1012-1017.

3 Maestrini I, Ducroquet A, Moulin S, Leys D, Cordonnier C, Bordet R: Blood biomarkers in the early stage of cerebral ischemia. Rev Neurol (Paris) 2016;172:198-219.

4 The Cochrane Collaboration. Review Manager (RevMan). Version 5.3 ed. Copenhagen: The Nordic Cochrane Centre, 2014. 
5 Moher D, Liberati A, Tetzlaff J, Altman DG; PRISMA Group: Preferred reporting items for systematic reviews and meta-analyses: the PRISMA statement. Ann Intern Med 2009; 151:264-269.

6 Leira R, Davalos A, Silva Y, Gil-Peralta A, Tejada J, Garcia M, et al: Early neurologic deterioration in intracerebral hemorrhage: predictors and associated factors. Neurology. 2004;63:461-467.

7 Arenillas JF, Rovira A, Molina CA, Grive E, Montaner J, Alvarez-Sabin J: Prediction of early neurological deterioration using diffusion - and perfusion-weighted imaging in hyperacute middle cerebral artery ischemic stroke. Stroke 2002;33:2197-2203.

8 Audebert HJ, Pellkofer TS, Wimmer ML, Haberl RL: Progression in lacunar stroke is related to elevated acute phase parameters. Eur Neurol 2004;51:125-131.

9 Bhatia K, Mohanty S, Tripathi BK, Gupta B, Mittal MK: Predictors of early neurological deterioration in patients with acute ischaemic stroke with special reference to blood urea nitrogen $(\mathrm{BUN}) /$ creatinine ratio \& urine specific gravity. Indian J Med Res 2015;141:299-307.

10 Bugnicourt JM, Roussel B, Garcia PY, Canaple S, Lamy C, Godefroy O: Aspirin non-responder status and early neurological deterioration: a prospective study. Clin Neurol Neurosurg 2011;113:196-201.

11 Campos F, Rodriguez-Yanez M, Castellanos M, Arias S, Perez-Mato M, Sobrino T, et al: Blood levels of glutamate oxaloacetate transaminase are more strongly associated with good outcome in acute ischaemic stroke than glutamate pyruvate transaminase levels. Clin Sci (Lond) 2011;121:11-17.

12 Cho BH, Kim JT, Chang J, Choi KH, Nam TS, Choi SM, et al: Early clinical implications of microalbuminuria in patients with acute ischaemic stroke. Postgrad Med J 2012;88:632638.

13 Chung JW, Kim N, Kang J, Park SH, Kim WJ, Ko Y, et al: Blood pressure variability and the development of early neurological deterioration following acute ischemic stroke. J Hypertens 2015;33:2099-2106.

14 Cuadrado-Godia E, Jimena S, Ois A, Rodriguez-Campello A, Giralt-Steinhauer E, Soriano-Tarraga C, et al: Factors associated with early outcome in patients with large-vessel carotid strokes. J Neurol Neurosurg Psychiatry 2013;84:305-309.

15 Davalos A, Toni D, Iweins F, Lesaffre E, Bastianello S, Castillo J: Neurological deterioration in acute ischemic stroke: potential predictors and associated factors in the European cooperative acute stroke study (ECASS) I. Stroke 1999;30:2631-2636.

16 Davalos A, Castillo J, Marrugat J, FernandezReal JM, Armengou A, Cacabelos P, et al: Body iron stores and early neurologic deterioration in acute cerebral infarction. Neurology 2000;54:1568-1574.

17 Davalos A, Castillo J, Pumar JM, Noya M: Body temperature and fibrinogen are related to early neurological deterioration in acute ischemic stroke. Cerebrovasc Dis 1997;7:6469.

18 Kanamaru T, Suda S, Muraga K, Okubo S, Watanabe Y, Tsuruoka S, et al: Albuminuria Predicts Early Neurological Deterioration in Patients with Acute Ischemic Stroke. Stroke. Los Angeles, American Heart Association/ American Stroke Association 2016 International Stroke Conference and State-of-theScience Stroke Nursing Symposium, 2017. Conference Start: 20160216. Conference End: 9. Conference Publication: (var.pagings). 47 (no pagination).

19 Kim JT, Kim HJ, Yoo SH, Park MS, Kwon SU, $\mathrm{Cho} \mathrm{KH}$, et al: MRI findings may predict early neurologic deterioration in acute minor stroke or transient ischemic attack due to intracranial atherosclerosis. Eur Neurol 2010; 64:95-100.

20 Kim JM, Moon J, Ahn SW, Shin HW, Jung $\mathrm{KH}$, Park KY: The etiologies of early neurological deterioration after thrombolysis and risk factors of ischemia progression. J Stroke Cerebrovasc Dis 2016;25:383-388.

21 Krarup LH, Sandset EC, Sandset PM, Berge E: $\mathrm{D}$-dimer levels and stroke progression in patients with acute ischemic stroke and atrial fibrillation. Acta Neurol Scand 2011;124:4044.

22 Kwan J, Hand P: Early neurological deterioration in acute stroke: clinical characteristics and impact on outcome. QJM 2006;99:625633.

23 Kwon HM, Lee YS, Bae HJ, Kang DW: Homocysteine as a predictor of early neurological deterioration in acute ischemic stroke. Stroke 2014;45:871-873.

24 Leira R, Davalos A, Aneiros A, Serena J, Pumar JM, Castillo J: Headache as a surrogate marker of the molecular mechanisms implicated in progressing stroke. Cephalalgia 2002; 22:303-308.

25 Lin LC, Fann WC, Chou MH, Chen HW, Su YC, Chen JC: Urine specific gravity as a predictor of early neurological deterioration in acute ischemic stroke. Medical Hypotheses 2011;77:11-14

26 Lin LC, Yang JT, Weng HH, Hsiao CT, Lai SL, Fann WC: Predictors of early clinical deterioration after acute ischemic stroke. Am J Emerg Med 2011;29:577-581.

27 Lorenzano S, Li H, Muzikansky A, Rost N, Batista L, Chutinet A, et al: Hyperacute Matrix Metalloproteinase-2 Levels Predict Early Neurological Deterioration in Acute Ischemic Stroke. Stroke. New Orleans, International Stroke Conference and Nursing Symposium, 2012. Conference Start: 20120201. Conference End: 3. Conference Publication: (var.pagings). 43 (2 Meeting Abstracts) (no pagination).

28 Matsumoto N, Kimura K, Yokota C, Yonemura K, Wada K, Uchino M, et al: Early neurological deterioration represents recurrent attack in acute small non-lacunar stroke. J Neurol Sci 2004;217:151-155.
29 Miyamoto N, Tanaka Y, Ueno Y, Kawamura M, Shimada Y, Tanaka R, et al: Demographic, clinical, and radiologic predictors of neurologic deterioration in patients with acute ischemic stroke. J Stroke Cerebrovasc Dis 2013; 22:205-210.

30 Mori M, Naganuma M, Okada Y, Hasegawa Y, Shiokawa Y, Nakagawara J, et al: Early neurological deterioration within 24 hours after intravenous rt-PA therapy for stroke patients: the stroke acute management with urgent risk factor assessment and improvement rt-PA registry. Cerebrovasc Dis 2012;34:140146.

31 Nacu A, Bringeland GH, Khanevski A, Thomassen L, Waje-Andreassen U, Naess H: Early neurological worsening in acute ischaemic stroke patients. Acta Neurol Scand 2016;133: 25-29.

32 Ogata T, Yasaka M, Wakugawa Y, Ibayashi S, Okada Y: Predisposing factors for acute deterioration of minor ischemic stroke. J Neurol Sci 2009;287:147-150

33 Saqqur M, Molina CA, Salam A, Siddiqui M, Ribo M, Uchino K, et al: Clinical deterioration after intravenous recombinant tissue plasminogen activator treatment: a multicenter transcranial Doppler study. Stroke 2007;38: 69-74.

34 Seners P, Turc G, Tisserand M, Legrand L, Labeyrie MA, Calvet D, et al: Unexplained early neurological deterioration after intravenous thrombolysis: incidence, predictors, and associated factors. Stroke 2014;45:2004-2009.

35 Seo WK, Seok HY, Kim JH, Park MH, Yu SW, $\mathrm{Oh} \mathrm{K}$, et al: C-reactive protein is a predictor of early neurologic deterioration in acute ischemic stroke. J Stroke Cerebrovasc Dis 2012;21:181-186.

36 Wang JY, Zhou DH, Li J, Zhang M, Deng J, Gao C, et al: Association of soluble intercellular adhesion molecule 1 with neurological deterioration of ischemic stroke: The Chongqing Stroke Study. Cerebrovasc Dis 2006;21: 67-73.

37 Cai JY, Lu C, Chen MH, Ba HJ, Chen XD, Lin $\mathrm{JH}$, et al: Predictive value of phosphorylated axonal neurofilament subunit $\mathrm{H}$ for clinical outcome in patients with acute intracerebral hemorrhage. Clin Chim Acta 2013;424:182186.

38 Shimoyama T, Kimura K, Uemura J, Saji N, Shibazaki K: Elevated glucose level adversely affects infarct volume growth and neurological deterioration in non-diabetic stroke patients, but not diabetic stroke patients. Eur J Neurol 2014;21:402-410.

39 Cha JK, Jeong MH, Kim EK, Lim YJ, Ha BR, $\mathrm{Kim} \mathrm{SH}$, et al: Surface expression of P-selectin on platelets is related with clinical worsening in acute ischemic stroke. J Korean Med Sci 2002;17:811-816

40 Bharosay A, Bharosay VV, Bandyopadhyay D, Sodani A, Varma M, Baruah H: Effect of lipid profile upon prognosis in ischemic and haemorrhagic cerebrovascular stroke. Indian J Clin Biochem 2014;29:372-376. 
41 Choi KH, Park MS, Kim JT, Chang J, Nam TS, Choi SM, et al: Serum triglyceride level is an important predictor of early prognosis in patients with acute ischemic stroke. J Neurol Sci 2012;319:111-116.

42 Kim YD, Choi HY, Jung YH, Yoo J, Nam HS, Song $\mathrm{D}$, et al: The ischemic stroke predictive risk score predicts early neurological deterioration. J Stroke Cerebrovasc Dis 2016;25:819824.

43 Kuo CY, Lin CH, Kuo YW, Hsu HL, Lin YH $\mathrm{Wu}$ CY, et al: Factor VIII levels are associated with ischemic stroke, stroke subtypes and neurological worsening. Curr Neurovasc Res 2015; 12:85-90

44 Lin CH, Kuo YW, Kuo CY, Hsu CY, Hsu HL, Lin $\mathrm{YH}$, et al: Shortened activated partial thromboplastin time is associated with acute ischemic stroke, stroke severity, and neurological worsening. J Stroke Cerebrovasc Dis 2015;24:2270-2276.

45 Zhang X, Sun Z, Ding C, Tang Y, Jiang X, Xie $\mathrm{Y}$, et al: Metabolic syndrome augments the risk of early neurological deterioration in acute ischemic stroke patients independent of inflammatory mediators: a hospital-based prospective study. Oxid Med Cell Longev 2016;2016:8346301

46 Wang X, Dong Y, Qi X, Huang C, Hou L: Cholesterol Levels and Risk of Hemorrhagic Stroke: a systematic review and meta-analysis. Stroke 2013;44:1833-1839.

47 Goldstein LB, Amarenco P, Szarek M, Callahan A 3rd, Hennerici M, Sillesen $\mathrm{H}$, et al: Hemorrhagic stroke in the stroke prevention by aggressive reduction in cholesterol levels study. Neurology 2008;70(24 pt 2):23642370.

48 Dziedzic T, Slowik A, Szczudlik A: Serum albumin level as a predictor of ischemic stroke outcome. Stroke 2004;35:e156-e158.

49 Brouns R, Wauters A, De Surgeloose D, Marien P, De Deyn PP: Biochemical markers for blood-brain barrier dysfunction in acute ischemic stroke correlate with evolution and outcome. Eur Neurol 2011;65:23-31.

50 Deb P, Sharma S, Hassan KM: Pathophysiologic mechanisms of acute ischemic stroke: an overview with emphasis on therapeutic significance beyond thrombolysis. Pathophysiology 2010;17:197-218.
51 Ramos-Cabrer P, Campos F, Sobrino T, Castillo J: Targeting the Ischemic Penumbra. Stroke 2010;42(1 suppl):S7-S11.

52 Castillo J, Davalos A, Noya M: Progression of ischaemic stroke and excitotoxic aminoacids. Lancet 1997;349:79-83.

53 Serena J, Leira R, Castillo J, Pumar JM, Castellanos M, Davalos A: Neurological deterioration in acute lacunar infarctions: the role of excitatory and inhibitory neurotransmitters. Stroke 2001;32:1154-1161.

54 Suda S, Katsumata T, Okubo S, Kanamaru T, Suzuki K, Watanabe Y, et al: Low serum n-3 polyunsaturated fatty acid/n-6 polyunsaturated fatty acid ratio predicts neurological deterioration in Japanese patients with acute ischemic stroke. Cerebrovasc Dis 2013;36: 388-393.

55 Zhou Y, Han W, Gong D, Man C, Fan Y: HsCRP in stroke: a meta-analysis. Clin Chim Acta 2016;453:21-27.

56 Topakian R, Strasak AM, Nussbaumer K, Haring HP, Aichner FT: Prognostic value of admission C-reactive protein in stroke patients undergoing iv thrombolysis. J Neurol 2008;255:1190-1196.

57 Vila N, Castillo J, Davalos A, Esteve A, Planas AM, Chamorro A: Levels of anti-inflammatory cytokines and neurological worsening in acute ischemic stroke. Stroke 2003;34:671-675.

58 Ovesen C, Christensen AF, Havsteen I, Krarup Hansen C, Rosenbaum S, Kurt E, et al: Prediction and prognostication of neurological deterioration in patients with acute $\mathrm{ICH}$ : a hospital-based cohort study. BMJ Open 2015; 5:e008563

59 Bellolio MF, Gilmore RM, Ganti L: Insulin for glycaemic control in acute ischaemic stroke. CochraneDatabaseSyst Rev2014;1:CD005346.

60 Alawneh JA, Moustafa RR, Baron JC: Hemodynamic factors and perfusion abnormalities in early neurological deterioration. Stroke 2009; 40:e443-e450

61 Nogueira RG, Jadhav AP, Haussen DC, Bonafe A, Budzik RF, Bhuva P, et al: Thrombectomy 6 to 24 hours after stroke with a mismatch between deficit and infarct. N Engl J Med 2018;378:11-21.

62 Kelly PJ, Murphy S, Coveney S, Purroy F, Lemmens R, Tsivgoulis G, et al: Anti-inflammatory approaches to ischaemic stroke pre- vention. J Neurol Neurosurg Psychiatry 2018; 89:211-218.

63 Bustamante A, López-Cancio E, Pich S, Penalba A, Giralt D, García-Berrocoso T, et al: Blood biomarkers for the early diagnosis of stroke. The stroke-chip study. Stroke 2017;48: 2419-2425.

64 Rozanski M, Waldschmidt C, Kunz A, Grittner U, Ebinger M, Wendt M, et al: Glial fibrillary acidic protein for prehospital diagnosis of intracerebral hemorrhage. Cerebrovasc Dis 2017;43:76-81.

65 Kelly PJ, Murphy S, Coveney S, Purroy F, Lemmens R, Tsivgoulis G, et al: Anti-inflammatory approaches to ischaemic stroke prevention. J Neurol Neurosurg Psychiatry 2018; 89:211-218.

66 Barber PA, Demchuk AM, Zhang J, Buchan AM: Validity and reliability of a quantitative computed tomography score in predicting outcome of hyperacute stroke before thrombolytic therapy. ASPECTS study group. Alberta stroke programme early CT score. Lancet 2000;355:1670-1674.

67 Maestrini I, Ducroquet A, Moulin S, Leys D, Cordonnier C, Bordet R: Blood biomarkers in the early stage of cerebral ischemia. Rev Neurol (Paris) 2016;172:198-219.

68 Khalil M, Teunissen CE, Otto M, Piehl F, Sormani MP, Gattringer T, et al: Neurofilaments as biomarkers in neurological disorders. Nat Rev Neurol 2018;14:577-589.

69 Shahim P, Gren M, Liman V, Andreasson U, Norgren N, Tegner Y, et al: Serum neurofilament light protein predicts clinical outcome in traumatic brain injury. Sci Rep 2016;6: 36791.

70 Gattringer T, Pinter D, Enzinger C, SeifertHeld T, Kneihsl M, Fandler S, et al: Serum neurofilament light is sensitive to active cerebral small vessel disease. Neurology 2017;89: 2108-2114.

71 Tiedt S, Duering M, Barro C, Kaya AG, Boeck J, Bode FJ, et al: Serum neurofilament light: a biomarker of neuroaxonal injury after ischemic stroke. Neurology 2018;91:e1338-e1347.

72 De Marchis GM, Katan M, Barro C, Fladt J, Traenka C, Seiffge DJ, et al: Serum neurofilament light chain in patients with acute cerebrovascular events. Eur J Neurol 2018;25: 562-568. 